\title{
Computer Modeling of Fast Collisionless Reconnection
}

\author{
J. N. LEBOEUF, F. BRUNEL, AND T. TAJIMA \\ INSTITUTE FOR FUSION STUDIES \\ UNIVERSITY OF TEXAS \\ AUSTIN, TEXAS 78712 \\ J . SAKAI \\ DEPARTMENT OF APPLIED MATHEMATICS \\ AND PHYSICS, FACULTY OF ENGINEERING \\ TOY AMA UNIVERSITY \\ TOYAMA 930 , J APAN
}

\author{
C. C. WU, AND J . M. DAWSON \\ DEPARTMENT OF PHYSICS \\ UNIVERSITY OF CALIFORNIA \\ LOS ANGELES, CALIFORNIA 90024
}

\begin{abstract}
ABS TRACT
Particle simulations of collisionless tearing, reconnection and coalescence of magnetic fields for a sheet-pinch configuration show that reconnection is Sweet-Parker like in the tearing and island formation phase. It is much faster to explosive in the island coalescence stage. Island coalescence is the most energetic process and leads to large ion temperature increase and oscillations in the merged state. Similar phenomena have been observed in equivalent MHD simulations. Coalescence and its effects, as observed in our simulations, may explain many of the features of solar flares and coronal X-ray brightening.
\end{abstract}

\section{INTRODUCTION}

Computer modeling of magnetic field reconnection, including island coalescence, has been tackled mainly with collisional MHD codes. Few kinetic studies of reconnection have been reported so far (Dickman and Morse, 1969; Amano and Tsuda, 1977; Katanuma and Kamimura, 1980; Terasawa, 1981; Hamilton and Eastwood, 1982; Leboeuf, Tajima and Dawson, 1981 and 1982; Tajima, 1982) even though in space plasmas collisionless tearing modes are believed to be one of the most effective mechanisms for magnetic field line reconnection. This is particularly true for the Earth's magnetospheric tail where the particles' mean free path is very large. Dickman, Morse and Nielson (1969) used a magnetostatic code to study tearing modes in the Astron fusion device. They found that the Astron plasma layer first develops tearing modes, but at later times the wavelength of this mode increases by coalescence until the plasma is completely reassambled. Amano and Tsuda (1977) were the first to study forced reconnection 
with an electromagnetic code. They forced a flow towards the initial magnetic neutral sheet and observed the formation of an $\mathrm{x}$-point. They also remarked that Joule heating in the diffusion region was not sufficient for the explosive energy release such as that observed in astrophysical and geophysical phenomena. The study of Katanuma and Kamimura (1980) involves using a magnetostatic code to study the nonlinear evolution of collisionless tearing modes. They verified the Drake-Lee (1977) theory of tearing. They did observe island coalescence in the case of multi-mode tearing. However, no discussion of the energetics of the interaction was given. Again using a magnetostatic code, Hamilton and Eastwood (1982) realistically modeled the geomagnetic tail and confirmed the stabilizing influence of small magnetic field normal to the sheet of the tearing mode. Finally, Terasawa (1981) used a reduced Darwin model, with electrostatics neglected, and followed the ions only. He verified the explosive tearing mode theory of Galeev, Coroniti and Ashour-Abdalla (1978). The question is whether adding the electrons and the electrostatics will modify his conclusions.

Our program of kinetic simulations of collisionless reconnection was primarily motivated by the laboratory experiments of W. Gekelman and R. L. Stenzel. (Stenzel and Gekelman, 1979; Gekelman and Stenzel, 1981) The tools used consist of magnetostatic and electromagnetic finite size particle simulation models with two spatial dimensions only. This paper will mainly be a review of our own work, and some comparisons with similar MHD simulations. Applications to the physics of solar flares will also be discussed.

By having a current flow in two strips perpendicular to the plane of the simulation, with the current ramped in time much as it is in the experiments of Gekelman and Stenzel, we are able to pass through the successive stages of current sheet formation in between the two strips. tearing of the current sheet to form magnetic islands and finally magnetic island coalescence.

The onset of coalescence occurs in an explosive fashion. Recent MHD simulations of reconnection and coalescence (Brunel, Tajima, and Dawson, 1982) exhibit comparable behaviour. Addition of a magnetic field parallel to the strips (a toroidal field in tokamaks) introduces incompressibility and prevents the fast reconnection that otherwise occurs (Tajima, 1982). The tearing phase leads to almost no energization of the particles' kinetic energy. Magnetic island coalescence, on the other hand, leads to a large increase in ion temperature. The oscillations exhibited by the ion temperature in the merged phase have also been observed in an MHD simulation of island coalescence ( $\mathrm{Wu}$, Leboeuf, Tajima, Dawson, 1980). These oscillations resemble what is reported of the solar gamma ray amplitude oscillations (Forrest et al., 1982) associated with loop coalescence in solar flares (Tajima, Brunel, Sakai, 1982).

\section{COMPUTER MODEL}

Our collisionless particle simulations follow the evolution of a plasma configration which is unstable against the tearing and subsequent coalescence instabilities. The electromagnetic code (Leboeuf, Tajima, Dawson, 1982) is two-and-one-half (two space, $\mathrm{x}$ and $\mathrm{y}$, and three velocity and field, $\mathrm{x}, \mathrm{y}$, and $\mathrm{z}$ dimensions) dimensional and periodic in both the particles and field quantities. The model includes electrons and ions, with ion-to-electron mass and temperature ratios of $\mathrm{M}_{\mathrm{i}} / \mathrm{m}_{\mathrm{e}}=10$ and $\mathrm{T}_{\mathrm{i}} / \mathrm{T}_{\mathrm{e}}=1 / 2$ respectively in a benchmark case. Both species of particles are given Maxwellian velocity distributions in all three directions at time $t=0$. Four 


\section{J. N. LEBOEUF, F. BRUNEL, T. TAJIMA, J. SAKAI, C. C. WU, J. M. DAW SON: \\ Computer Modling of Fast Collisionless Reconnection}

particles per unit cell are typically used. The particles are loaded uniformly in space so that the density is uniform at $\mathrm{t}=0$. We focus on rectangular system sizes $\mathrm{L}_{\mathrm{x}} \times \mathrm{L}_{\mathrm{y}}=128 \Delta \times 32 \Delta$ and $256 \Delta \times 16 \Delta$, where $\Delta$ is the unit grid spacing. For the first, $\Delta=\lambda_{\mathrm{e}}$, the electronic Debye length and the speed of light $\mathrm{c}=\mathrm{v}_{\mathrm{te}}$, the electron thermal velocity, so that the collisionless skin depth $\delta=\mathrm{c} / \omega_{\mathrm{Pe}}=5 \lambda_{\mathrm{e}}$. For the second, $\Delta=2 \lambda_{\mathrm{e}}$, with collisionless skin depth $\mathrm{c} / \omega_{\mathrm{pe}}=6 \lambda_{\mathrm{e}}$ and the ratio of thermal velocity to speed of light $v_{t e} / c=1 / 6$.

The magnetic configuration is established by extemal current strips placed at $\mathrm{y}=0$ and $\mathrm{y}=\mathrm{Ly}$ which extend along $\mathrm{x}$. The current flows in the $\mathrm{z}$-direction. To avoid infinite magnetic energy, the retum path of the current is chosen to be through the plasma. At $t=0$, the current is zero in the strips and rises sinusoidally from zero at $t=0$ to a maximum at the quarter period after which it is kept constant (crowbar phase), with a rise time greater than or equal to the magnetosonic transit time from center to plates. By varying the strength of the currents in the strips, different plasmas are obtained. For the $128 \times 32$ case, the plasma $\beta=0.2$, for maximum magnetic field and average density. This entails electron and ion Larmor radii of $1.3 \lambda_{\mathrm{e}}$ and $2.6 \lambda_{\mathrm{e}}$ respectively, and an Alfvén velocity $\mathrm{v}_{\mathrm{A}}=1.22 \mathrm{v}$ te. For the $256 \times 16$ case, the maximum field is such that $\beta=0.06, \rho_{\mathrm{e}}=.85 \lambda_{\mathrm{e}}, \rho_{\mathrm{i}}=1.90 \lambda_{\mathrm{e}}$. The Alfvén speed is $\mathrm{v}_{\mathrm{A}}=2.2$ $\mathrm{v}_{\text {te }}$. When a constant toroidal field is imposed in the $\mathrm{z}$-direction it is such that $0.2 \lesssim \mathrm{B}_{\mathrm{t}} / \mathrm{B}_{\mathrm{p}} \lesssim$ 4 where $B_{p}$ is the maximum poloidal field. Straight forward calculations of the magnetic Reynolds number from the finite size particles collision frequency yield $S=800$. However since the current flow is in the ignorable $z$ direction, the actual collisionality may be regarded as much smaller than the in-plane value and $\mathrm{S}$ greater than the above value.

It should be noted that our model is limited by the periodic boundary con litions imposed on both the particles and fields. Moreover, the system sizes used only describe a few collisonless skin depths, ion Larmor radii and Debye lengths. We are actually looking at rather microscopic $\mathrm{x}$-points and o-points.

\section{MAGNETIC RECONNECTION}

We discuss the compressible cases without toroidal field first and those with toroidal field next. The "collisionless" reconnection rate in both cases is measured and compared with theory and similar MHD simulations.
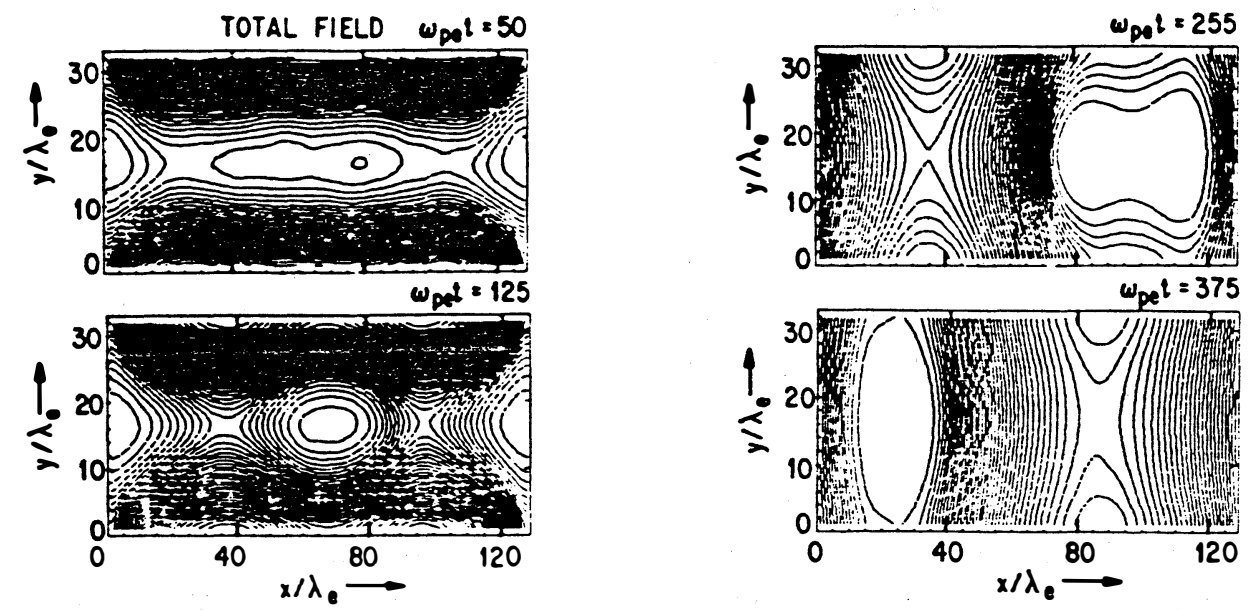

Fig. 1 Time evolution of the magnetic field lines in the $128 \times 32$ case with $B_{t} / B_{p}=0$. (Leboeuf, Tajima, Dawson, 1982) 


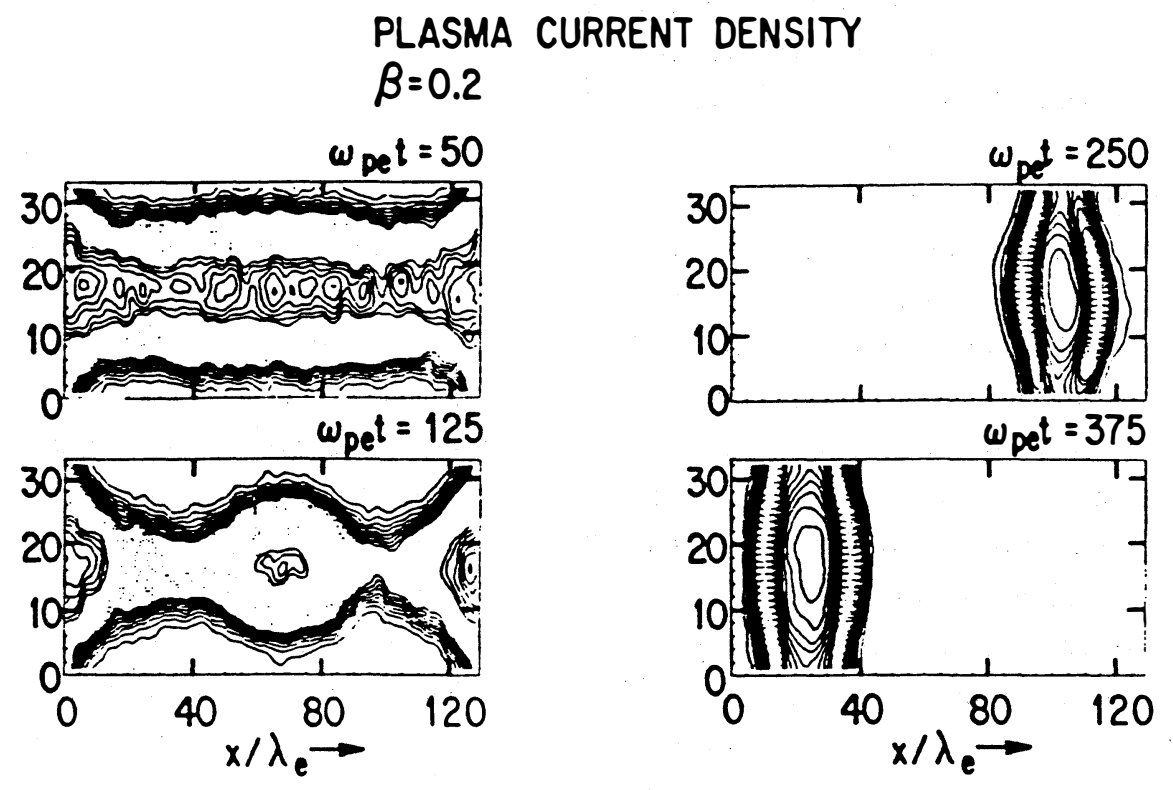

Fig. 2 Time evolution of the current density in the $128 \times 32$ case with $B_{t} / B_{p}=0$. The dotted contours indicate regions of maximum plasma current. (Leboeuf, Tajima, Dawson, 1982)

The evolution of the field lines in the $128 \times 32$ case $\left(B_{t}=0\right)$ is shown in Fig. 1. The corresponding plasma current density is displayed in $\mathrm{F}$ ig. 2 . The various stages of evolution consist of a current sheet formation at the center of the system, break-up of the current sheet to form a chain of $\mathrm{x}$-points and $\mathrm{o}$-points, i.e. establish a sheet pinch configuration, swelling of the islands by reconnection and finally coalescence of the islands. All of these phenomena happen within 2 to 4 Alfvén times. As the current increases in the strips, an equivalent amount of current is returned through the plasma. (Note the external current is maintained throughout the simulations.) Pinching occurs and induces a flow $\left(\mathrm{V}_{\mathbf{y}}= \pm \mathrm{J}_{\mathrm{p}}^{z} \times \mathrm{B}_{\mathrm{x}}^{\mathrm{e}}\right)$ through the $\mathrm{x}$-points and into the o-points where the plasma remains trapped. The attractive force between the so-formed plasma filaments induces coarescence. The external circuit is coupled to the plasma and is the source of free energy. The events described are forced on the plasma by the external circuit and in that sense we are looking at forced reconnection.

In the $256 \times 16$ case $\left(B_{t}=0\right)$, the change in magnetic topology is best illustrated by the plasma current density. Tearing of the long and narrow current sheet induces 16 islets. They eventually coalesce pairwise down to one island, as shown in Fig. 3.

Tajima (1982) found that when a constant toroidal field $\mathrm{B}_{z}$ of strength $\mathrm{B}_{t}$ is added to the above configuration things happen in a qualitatively similar fashion for $\mathrm{B}_{\mathrm{t}} \lesssim \mathrm{B}_{\mathrm{p}}$, the maximum poloidal field, as when $\mathrm{B}_{\mathrm{t}}=0$ in terms of topological changes. In particular the violent coalescence instability is still seen. When $B_{t} \gtrsim B_{p}$, however, the current sheet still tears into many islets but the coalescence instability does not occur (within the simulated time scales). 


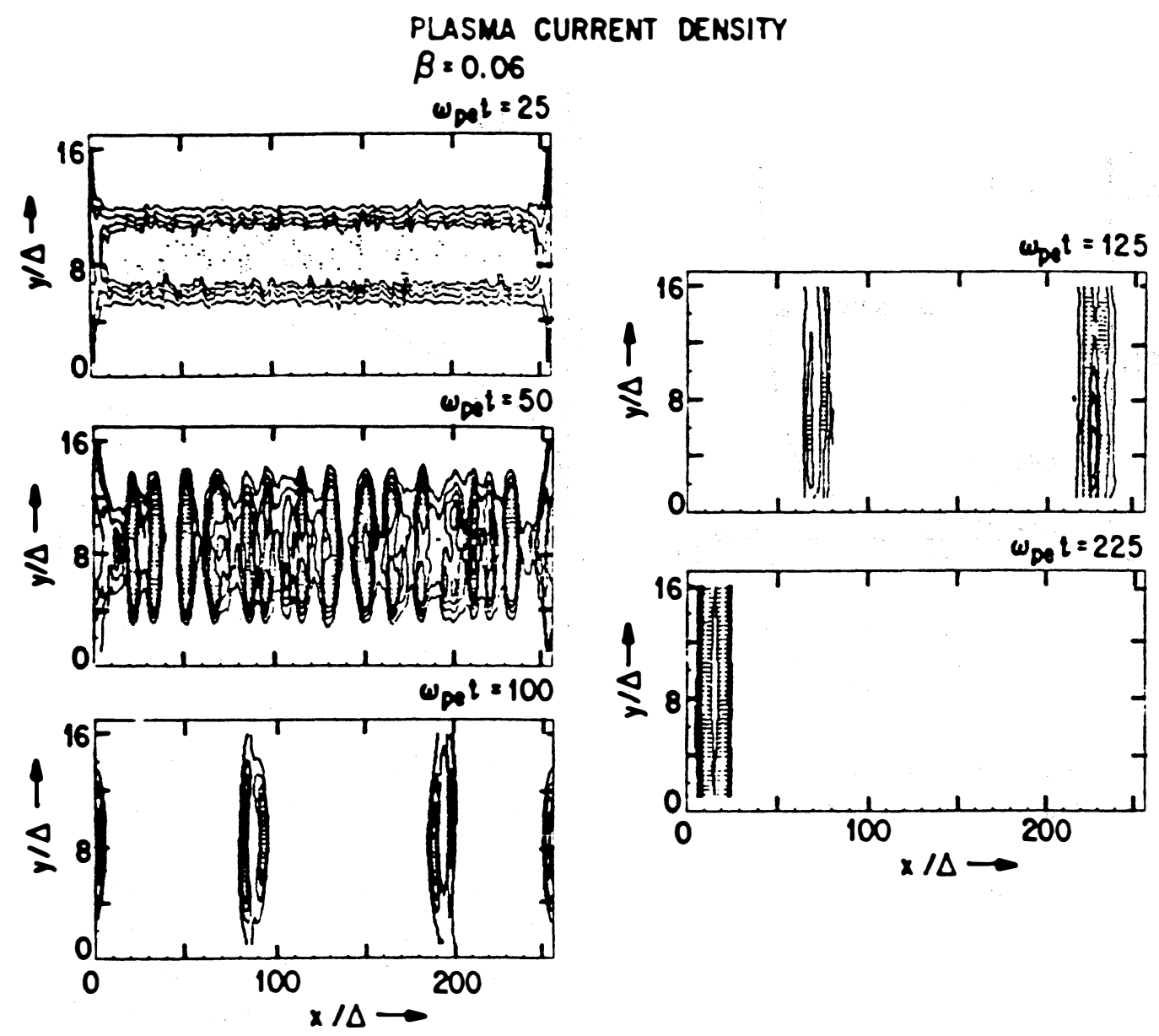

Fig. 3 Time evolution of the current denstty in the $256 \times 16$ case with $B_{t} / B_{p}=0$. (Leboeuf, Tajima, Dawson, 1982)

For $\mathrm{B}_{\mathrm{t}} \gtrsim \mathrm{B}_{\mathrm{p}}$ i.e. strong field perpendicular to the plane of the simulations, the plasma is strongly magnetized $\left(\rho_{\mathrm{e}} \lesssim \Delta, \rho_{\mathrm{i}} \lesssim 3 \Delta\right)$ and cross-field motion of the particles is strongly impaired except through $\mathrm{E} \times \mathrm{B}$ spatial diffusion, i.e. the plasma is nearly incompressible since $\boldsymbol{\nabla}$. $\mathrm{v}=\boldsymbol{\nabla} \cdot(\mathrm{E} \times \mathrm{B})=0$. In this case many islets lead to turbulence of tearing modes. Each mode has a lifetime roughly the jnverse of the linear growth rate. A renormalized turbulence theory was constructed by Tajima (1982) based on this observation. The flux increases with a time exponent smaller than predicted by Rutherford (1973) or Drake and Lee (1977) and may be explained by this turbulent tearing mode theory. In the collisionless tearing turbulence the magnetic fluttering-induced electron response adds the essentially new physics.

Measurements of the poloidal flux trapped in one island or islet (private flux) as a function of time for $\mathrm{B}_{\mathrm{t}} / \mathrm{B}_{\mathrm{p}}=0.2(128 \times 32$ case $)$ and $\mathrm{B}_{\mathrm{t}} / \mathrm{B}_{\mathrm{p}}=2(256 \times 16$ case $)$ are shown in Figs. $4 \mathrm{a}$ and $4 \mathrm{~b}$ resspectively. In the weak toroidal field case, a linear phase, which encompasses the 

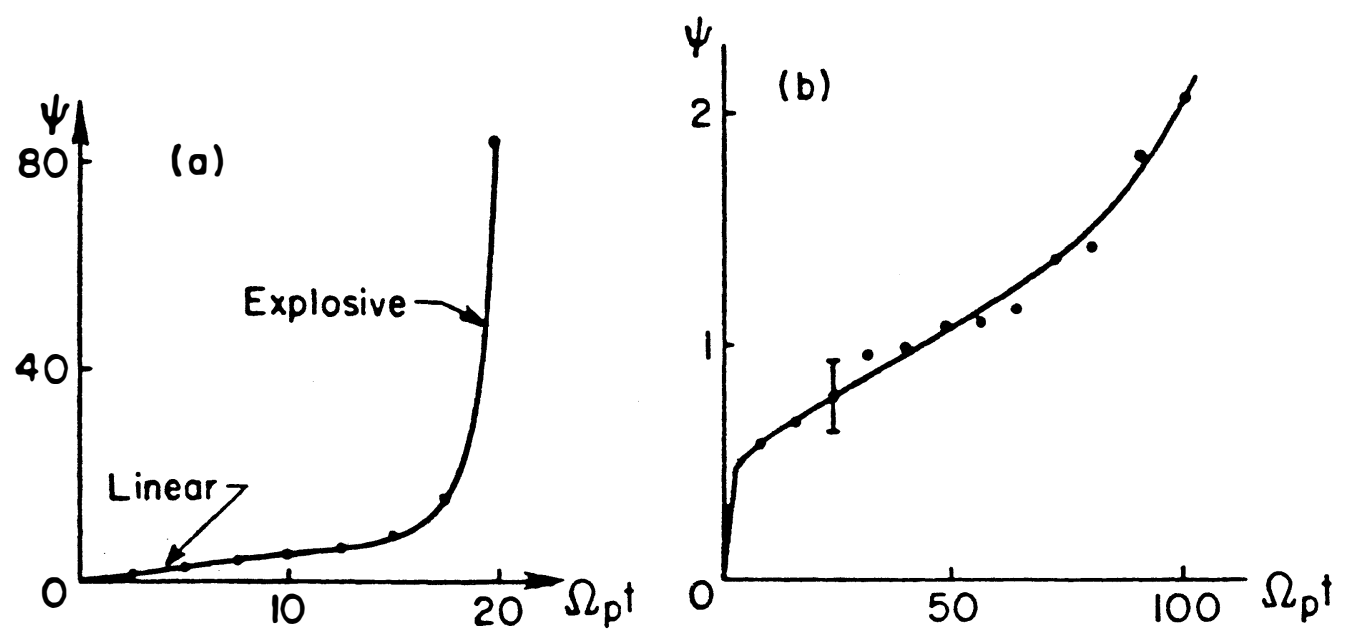

Fig. 4 Time evolution of the private poloidal flux in a) the $128 \times 32$ case with $B_{t} / B_{p}=0.2$ and b) in the $256 \times 16$ case with $\mathrm{B}_{\mathrm{t}} / \mathrm{B}_{\mathrm{p}}=2 . \quad \Omega_{\mathrm{p}}$ represents the electron gyrofrequency measured with respect to the maximum poloidal field. (Tajima, 1982)

island swelling stage, preceds the explosive coalescence one. For the strong toroidal field case, the rise is linear in time over ten times longer time scales and the flux increase is $10^{-2}$ times smaller.

MHD simulations of magnetic reconnection driven either by external currents pinching the plasma by Brunel, Tajima and Dawson (1982) or by the coalescence instability of an equilibrium chain of magnetic islands by Bhattacharjee, Brunel and Tajima (1983) show similar behavior. It is found that fast magnetic reconnection may consist of more than one stage. After the Sweet-Parker phase (Parker, 1979) is established for an Alfvén time, a faster second phase of reconnection takes over if the plasma is compressible. The Sweet-Parker flux is

$$
\psi_{\mathrm{sp}}(t)=\eta^{1 / 2} B_{\mathrm{p}}(y=a)\left(\frac{n_{\mathrm{i}}}{n_{\mathrm{e}}}\right)^{1 / 2}\left(\frac{v_{\mathrm{A}}}{L}\right)^{1 / 2} t,
$$

where $n_{e}$ and $n_{i}$ are the densities outside and inside the current channel, a the current channel width, $\eta$ the resistivity-, $\mathrm{L}$ the length of the reconnecting region, $\mathrm{B}_{\mathrm{p}}$, the poloidal field and $\mathrm{v}_{\mathrm{A}}$ the Alfvén velocity. Our basic equations to describe the system have been reduced in order to gain reasonable analytical expressions and straightforward understanding of the underlying physics:

(outside)

$$
\begin{aligned}
& \frac{\partial \psi}{\partial t}=v_{1} \times B_{\mathrm{p}}, \\
& p_{\mathrm{e}}^{2}+B_{\mathrm{e}}^{2} / 8 \pi=p_{\mathrm{i}}^{2}+B_{\mathrm{i}}^{2} / 8 \pi \sim p_{\mathrm{i}},
\end{aligned}
$$

(inside)

$$
\begin{aligned}
& \frac{\partial \psi}{\partial t}=\eta \nabla_{\perp}^{2} \psi \approx \eta B_{\mathrm{p}} / a \\
& n_{\mathrm{e}} L v_{\perp}=n_{\mathrm{i}} a u
\end{aligned}
$$




\section{J. N. LEBOEUF, F. BRUNEL, T. TAJIMA, J. SAKAI, C. C. WU, J. M. DAWSON: \\ Computer Modling of Fast Collisionless Reconnection}

The left-hand side of Eq. (5) is the particle flux outside the separatrix, while the right-hand side is that within the separatrix. The first equations (Eq. (2) and Eq. (4)) are the magnetic flux equations, i.e. Faraday's law, while the second equations (Eq. (3) and Eq. (5)) are related to the equations of motion of the plasma. As is expected, the outside solution should be the MHD solution according to Eq. (2). The velocity $\mathrm{u}$ in the intemal layer was determined to be

$$
u \sim v_{\mathrm{Ai}}=B_{\mathrm{e}} /\left(4 \pi M n_{\mathrm{i}}\right)^{1 / 2}
$$

by Brunel and Tajima (1983). Using Eqs. $(2)-(6)$ the second phase flux is calculated to go as

$$
\psi=\psi_{\mathrm{sp}}\left(t_{\mathrm{A}}\right)\left(t / t_{\mathrm{A}}\right)^{\mathrm{n}_{i} / \mathrm{n}_{e}} .
$$

Scalings of $\psi \propto t^{4}$ have been obtained in the pinching cases. Coalescence driven reconnection yields scalings up to $\psi \propto t^{2}$ for the compressible cases, but $\psi \propto \mathrm{t}$ when a large toroidal field is applied.

\section{ENERGETI CS}

We concentrate here on cases with $0 \lesssim \mathrm{B}_{\mathrm{t}} / \mathrm{B}_{\mathrm{p}} \lesssim 0.2$ or compressible situations. A summary of the behavior of the various components of the temperatures for the $128 \times 32$ case with $\mathrm{B}_{\mathrm{t}}=0$ is given in Fig. 5 .
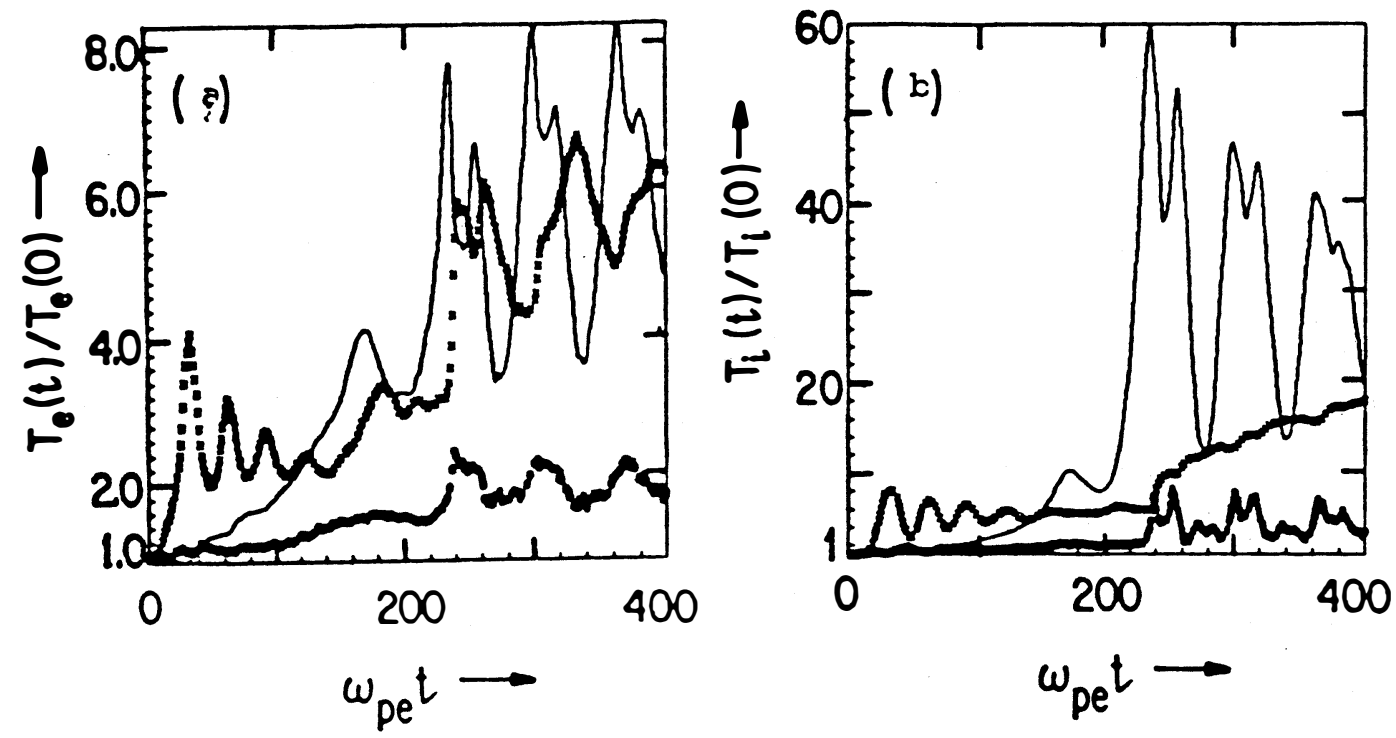

Fig. 5 Time evolution of the temperatures in all three directions in the $128 \times 32$ case with $\mathrm{B}_{\mathrm{t}} / \mathrm{B}_{\mathrm{p}}$ $=0$. for $\mathrm{a}$ ) electrons and $\mathrm{b}$ ) ions. The full curve refers to the $\mathrm{x}$-direction, the crosses and circles to the $y$ and $z$ ones respectivelv. (Leboeuf. Taima. Dawson. 1982)

No sizable increase is detected in the current sheet formation and tearing phases, just the adiabatic compression component associated with the external current rise in the strips. As the islands coalesce, fast increase in the temperatures is apparent. Most of the increase is concentrated in the $\mathrm{x}$-direction, where the ion temperature in the merged phase is on average 30 times 
its initial level. The ions achieve a higher temperature than the electrons in all three directions. Note the large oscillations in the ion and electron temperatures with a period $\tau=60 / \omega_{\mathrm{pe}}$ in the merged phase. The momentum distribution functions of electrons and ions in the $\mathrm{x}$-direction displayed in Fig. 6 exhibit bulk heating and symmetric high energy tails at late times. As shown in Fig. 7, an even more pronounced increase in temperature is obtained from the successive coalescence events of the $256 \times 16$ case with $\mathrm{B}_{\mathrm{t}}=0$.
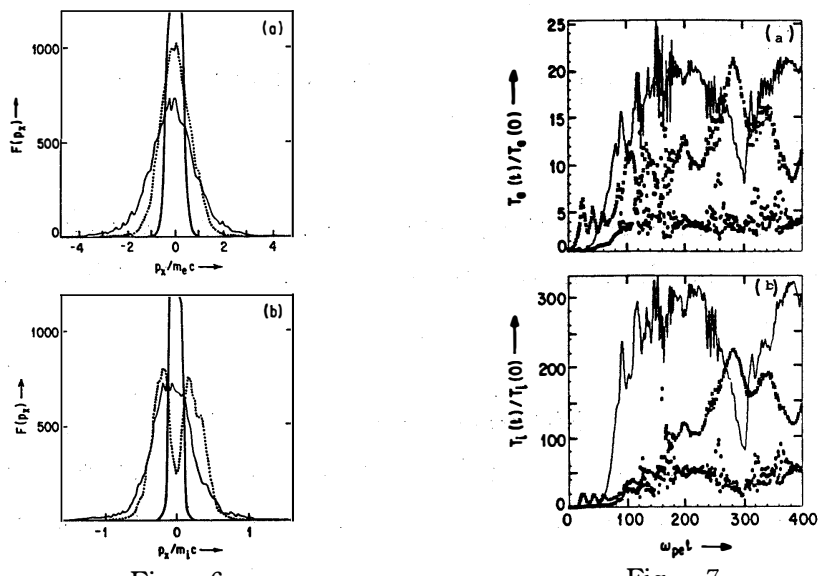

Fig. 7

Fig. 6 Momentum distribution functions in the $\mathrm{x}$-direction for the $128 \times 12$ case with $\mathrm{B}_{\mathrm{t}} / \mathrm{B}_{\mathrm{p}}$ $=0$. and for a) electrons and b) ions at $\omega_{\mathrm{pe}} \mathrm{t}=50$ (full bold curve: island formation stage), $\omega_{\text {pe }} \mathrm{t}=225$ (dotted curve: coalescence phase), $\omega_{\text {pe }} \mathrm{t}=325$ (full thin curve: merged qtate.). The momenta are normalized to $m_{e} c$ for the electrons and $M_{1} \quad c$ for the ions. (Leboeuf, Tajima, Daw son, 1982)

Fig. 7 Time evolutipn of the temperatures in all three directions in the $256 \times 16$ case with $\mathrm{B}_{\mathrm{t}}$ $/ B_{p}=0$. for $\left.a\right)$ el ectrons and $b$ ) ions. The full curve refers to the $x$-direction, the crosses and circles to the $y$ and $z$ ones respectively. (Leboeuf, Tajima, Dawson, 1982)

Results for the compressible $128 \times 32$ case with $\mathrm{B}_{\mathrm{t}} / \mathrm{B}_{\mathrm{p}}=0.2$ are displayed in Fig. 8. Fig. 8 a is a plot of ion temperature versus time. Again sharp increase upon coalescence followed by oscillations is apparent. Fig. $8 \mathrm{~b}$, which represents the ion ditribution function in the $\mathrm{x}$-direction after coalescence, shows bulk heating and symmetric tails.

Fig. $8 \mathrm{c}$ is a plot of the ion distribution function along the toroidal field. It shows three regimes. First the bulk, then the exponential section $\mathrm{f}_{2}\left(\mathrm{p}_{\mathrm{z}}\right)$ $=\exp \left(-\mathrm{P}_{\mathrm{z}} / \mathrm{P}_{0}\right)$
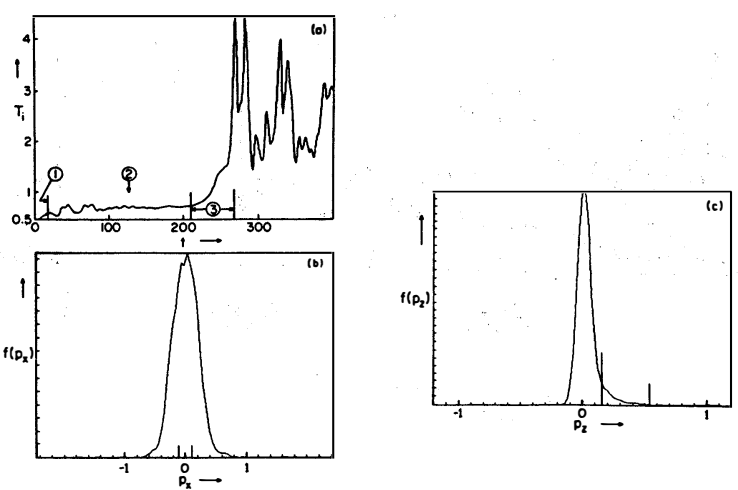

Fig. 8 Various data from $128 \times 32$ case with $B_{t} / B_{p}=0.2$. a) Time evolution of the ions temperature. Phases 1 and 2 are the tearing growth and saturation phases, phase 3 is the explosive coalescence phase. The period of temperature oscillations in the merged phase is $\tau \sim \tau$ A. b) ion distribution function in the $\mathrm{x}$-direction and in the merged state. c) I on distribution function in the $\mathrm{z}$-direction and in the merged state. The thermal momenta are indicated by tickmarks near $\mathrm{p}=\dot{0}$. Momenta are normalized with respect to $M_{i}$ c. (Tajima, Brunel, S akai, 1982) 


\section{J. N. LEBOEUF, F. BRUNEL, T. TAJIMA, J. SAKAI, C. C. WU, J. M. DAW SON:}

Computer Modling of Fast Collisionless Reconnection

and third the flat distribution extending up to the relativistic factor $\gamma \sim 2$ in the relativistic region, where $\mathrm{p}_{0}^{2} / 2 \mathrm{M}_{\mathrm{i}}=10 \mathrm{x}$ (bulk temperature). The bulk ion heating in the is attributed to the adiabatic heating" ; the exponential heating in the $\mathrm{x}$ and $z$ directions are inductive in nature. The hot flat long tails in the $\mathrm{x}$ and $\mathrm{z}$ directions are due to acceleration by the magnetosonic shock ( Tajima et al., 1983). The maximum energy may be estimated based on the Alfvén Mach uumber. Most of the energy of the particles still belongs to the bulk component, however.

We have seen that the total flux reconnection of two islands takes place within 1 to $2 \mathrm{Al}$ fvén times. The magnetic energy contained in the islands is explosively released into kinetic energy as seen in Figs. 6, 7 and 8 . The amount of available potential energy $\mathrm{W}_{\mathrm{c}}$ by attracting two toroidal current rods I of radius a with separation $\mathrm{L}$ is

$$
W_{\mathrm{c}} \simeq-2 I^{2} / c^{2} \ln (L / a) .
$$

Our simulations show that about $1 / 6$ of the energy $\mathrm{W}_{\mathrm{c}}$ was transferred to kinetic energy upon coalescence in the $128 \times 32$ case with $\mathrm{B}_{\mathrm{t}}=0$. This amount of energy conversion is about two magnitude above that during the tearing process. The oscillations in temperature observed in the $128 \times 32$ cases are found to be magnetosonic ones with a frequency $\omega=\mathrm{kv}_{\mathrm{A}}$, where $\mathrm{k}=2 \pi / \mathrm{a}, \mathrm{a}$ the current channel width and $\mathrm{v}_{\mathrm{A}}$ calculated according to the magnetic field measured at the island. These temperature oscillations can be attributed to the overshooting of the two coalescing and colliding current filaments. Once the two filaments merge, they are bound by the common flux and the resulting island shape oscillates from prolate to oblate on the time scale of the temperature oscillations. The colliding plasmas cause turbulent flows within the final island and the originally directed energy is eventually dissipated into heat. The turbulent mixing in the island also cause the bulk heating observed on the distribution functions.

Similar features have also been observed in 2-D Eulerian MHD simulations of the coalescence instability of magnetic islands formed by nonlinear tearing modes ( $\mathrm{Wu}$, Leboeuf, Tajima and Dawson, 1980). A sheet-pinch configuration is modeled. Initially small perturbations consisting of two linearized eigenfunctions are imposed: one with a wavelength $\lambda=\mathrm{L} / 2$ and a smaller perturbation with $\lambda=\mathrm{L}$, $\mathrm{L}$ being the system size. The perturbation with $\mathrm{L} / 2$ gives rise to the formation of two magnetic islands at the stage of the nonlinear tearing mode. These islands then interact with each other due to the presence of the smaller perturbation with wavelength $\lambda=\mathrm{L}$; they coalesce and merge into larger units. The time evolution of the fluid kinetic energy for an MHD case with $\mathrm{S}=2000$ is displayed in Fig. 9b, alongside a temperature plot for the $128 \times 32$ case with $B_{t}=0$, The initial increase in energy up to $t=120 \tau_{\mathrm{A}} \quad$ corresponds to the exponential growth of the $\mathrm{L} / 2$ perturbation with linear growth rate $\gamma_{\mathrm{L} / 2}=9.6$ $\times 10^{-3}$. By $\mathrm{t}=720 \tau_{\mathrm{A}}$, the perturbation with wavelength $\lambda=\mathrm{L}\left(\gamma_{\mathrm{L}}=1.2 \times 10^{-2}\right)$ has reached a large enough amplitude to trigger coalescence. During coalescence, the fluid energy rises almost exponentially with growth rate $\gamma_{C}=3.8 \times 10^{-2}\left(\gamma_{C}>\gamma_{L}, \gamma_{L / 2}\right)$. While the kinetic energy only accounted for $0.04 \%$ of the magnetic energy up to $t=720 \tau_{\mathrm{A}}$, it accounts for $10 \%$ of it in the merged phase, 220 times the saturation value for the island formation stage. In the merged phase, the kinetic energy presents oscillations whose priod $\tau=80 \tau_{\mathrm{A}}$, with $\tau_{\mathrm{A}}$ defined with respect to the asymptotic field, roughly equivalent to oscillations at the Alfvén frequency 

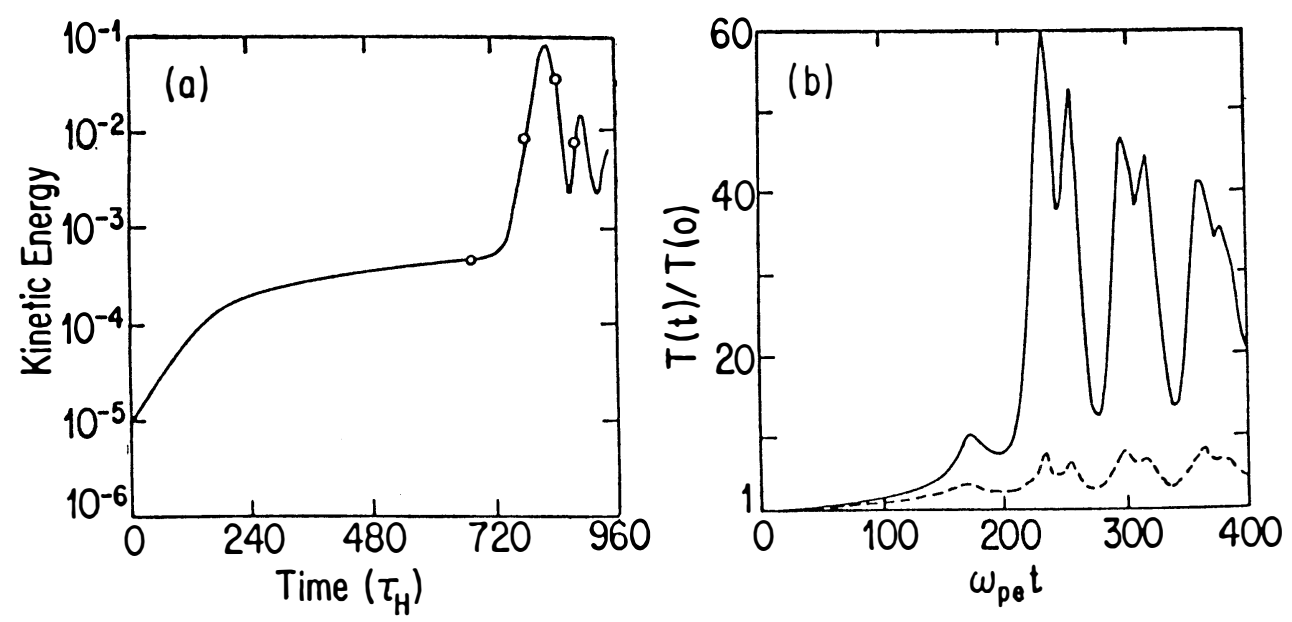

Fig. 9 Comparative data from particle simulations and MHD simulations. a) I on (full curve) and electron (dotted curve) temperatures in the $\mathrm{x}$-direction for the $128 \times 32$ case with $\mathrm{B}_{\mathrm{t}} / \mathrm{B}_{\mathrm{p}}=0$. polotted as a function of time. b) Time evolution of the kinetic energy for the MHD simulation of island coalescence induced by nonlinear tearing modes with $\mathrm{S}=2000$. Note the oscillations on both at $\tau \sim \tau_{\mathrm{A}}$, with the poloidal field measured at the island. ( $\mathrm{Wu}$, Leboeuf, Tajima- Dawson, 1982) ,

determincd with the field at the island. These oscillations and the larger energy gain upon coalescence are similar to what we observe in the particle simulations.

\section{APPLICATION TO SOLAR FLARES}

Recent direct observations in soft X-rays (Howard and Svetska, 1977) of ' interconngcting coronal loops spur the theorist to consider loop coalescence as an important process for solar flares and coronal X-ray brightening phenomena. Another recent observation (Forrest et al., 1982) of amplitude oscillations in gamma-ray emission from the impulsive phase of a solar flare adds curiosity and an important clue to the underlying physical process. The nonlinear development of the coalescence instability of the current loops might provide a coherent explanation of the above observations ( Tajima, Brunel and Sakai, 1982). Some of the results presented here offer a quantitative and natural explanation of such known characteristics as the impulsive nature of flares, the time scale of the impulsive phase, intense heating by flares, and formation of the high energy tails on the particle distributions.

The following scenario has been proposed by Tajima, Brunel and Sakai (1982). The flare loop slowly expands after it emerges from the photosphere as the toroidal field curvature of the loop makes the centrifugal motion. In time, the toroidal current $\mathrm{J}_{\mathrm{t}}$ builds up, increasing the poloidal magnetic field $B_{p}$. As the poloidal field $B_{p}$ reaches the critical value that is of the order of magnitude $\mathrm{B}_{\mathrm{t}}$, the adjacent flare current loops can now coalesce rapidly facilitated by the fast reconnection process governed by Eq. (7), the faster second phase. Such a fast coalescence of flare loops proceeds explosively once in its nonlinear regime in a matter of one or two Alfvén times, releasing more than one-tenth of the magnetic energy into (ion) kinetic energy. 


\section{J. N. LEBOEUF, F. BRUNEL, T. TAJI MA, J. SAKAI, C. C. WU, J . M. DAW SON: \\ Computer Modling of Fast Collisionless Reconnection}

For the flare loop magnetic field ( 100 Gauss) with current rod size $\left(\mathrm{a}=10^{8} \mathrm{~cm}\right)$, the energy density is $\mathrm{W}_{\mathrm{c}} \sim 0.5 \times 10^{20} \mathrm{\ell n}(\mathrm{L} / \mathrm{a}) \sim 1.5 \times 10^{20} \mathrm{erg} / \mathrm{cm}$ and the energy available in length $=\mathrm{L}\left(\sim 10^{9} \mathrm{~cm}\right)$ is $\mathrm{E}=1.5 \times 10^{29}$ erg for $\mathrm{a}=10^{8}, \mathrm{~d}=\mathrm{L}=10^{9}$ and $\mathrm{E}=1.5 \times 10^{31}$ erg for $\mathrm{a}=10^{9}$, $\mathrm{d}=\mathrm{L}=10^{10}$. The released ion energy is $\mathrm{E}_{\text {ion }} \sim \mathrm{E} / 6$ and is in between $2 \times 10^{28}$ and $2 \times 10^{30}$ erg due to coalescence. This amount of energy is in the neighborhood of the solar flare energy (Sturrock, 1980). These energies can be releasgd during the impulsive phase as well as during the main phase.

With this magnetic field, the Alfven time is of the order of $1-3 \mathrm{~s}$, which is approximately the time scale for fast coalescence. The time scale for the impulsive phase is observed to be of the order of a few seconds. The sudden nature of the impulsive flare phase (Sturrock, 1980) is thus explained by increasing the fiejd aligned current and by the faster secend phase reconnecti on in the course of coalescence. The field aligned particle distribution $f\left(p_{z}\right)$ of Fig. 8 should represent approximately the energy observed in gamma rays from the flare loop interface with the photosphere where the energetic pbrticles react with dense photospheric nuclei. The $\mathrm{X}$-ray spectra represent the electron energy distribution, which also shows the oscillatory characteristics in parallel with the ions characteristics. Observation of these radiation spectra by Chupp, Forrest and Suri (1975) shows that the soft X-ray energy domain (up to $400 \mathrm{keV}$ ) and hard X-ray domain ( up to $7 \mathrm{M} \mathrm{MeV}$ ) have different distribution characteristics: in the hard $\mathrm{X}$-ray domain $(700 \mathrm{keV}-7 \mathrm{MeV})$ the energy spectrum is exponential. This type of characteristic seems to match the simulation results of Fig. 8, where the particle distribution breaks into the bulk, the $\exp \left(-\mathrm{p}_{\mathrm{z}} / \mathrm{p}_{0}\right)$ domain (energy up to a typical temperature 10-50 times of the bulk temperature), and the flat low-population relativistic domain. The amplitude of the oscillation $(\sim 1$ Alfvén time) and its more minute characteristics, resemble what is reported of the solar gamma ray amplitude oscillations (Forrest et al., 1982).

\section{DISCUSSION}

We have examined through collisionless particle simulations some of the phenomena associated with current sheet formation, tearing of the sheet to form a chain of $\mathrm{x}$-points and $\mathrm{o}$-points and finally island coalescence. The analysis of these phenomena is far from complete. Nevertheless, the measured reconnection rates in the island formation stage can be matched with theoretical results from an analysis which is a modification of the Sweet-Parker reconnection rate by plasma compressibility. The effective resistivity is supplied in the collisionless case by wave - particle interactions and turbulent electron orbit modifications. The coalescence instability leads to an explosive increase of the reconnection rate. The consequences of tearing on the plasma are minimal in terms of particle energy gains. The consequences of the coalescence instability are large ion temperature increases and large temperature oscillations in the merged phase. The energy increase is accounted for by the loss of potential energy of the attracting current filaments, i.e. loss of poloidal magnetic energy. The oscillations in temperature are explained simply by the oscillations of the merged island at its magnetosonic frequency. It has been interesting to note that similar phenomena are observed in MHD simulations of reconnection and island coalescence even though both types of simulations cover vastly differing spatial scales. Finally, the nonlinear development of the coalescence instability seems to account for the im- 
pulsive nature of some types of solar flares, their time scale, intense plasma heating by flares and formation of high gnergy tails on the particle distributions.

\section{ACKNOWLEDMENTS}

This work was supported by the National Science Foundation grant ATM 82-14730 and Department of Energy, Office of Fusion Energy grant DE-FG05-80-ET-53088.

\section{REFERENCES}

Amano, K. , and T. Tsuda, Reconnection of magnetic field lines by clouds-in -cells plasma model, J. Geomag. Geoelectr. 29, 9, 1977.

Bhattacharjee, A., F. Brunel, and T. Tajima, Magnetic reconnection driven by the coalescence instability, Phys. Fluids 26, 3322, 1983.

Brunel, F., T. Tajima, and J. . M. Dawson, Fast magnetic reconnection processes, Phys. Rev. Letts. 49, 323, 1982.

Brunel, F., and T. Tajima, Confinement of a high -beta plasma column, Phys. Fluids 26, $535,1983$.

Chupp, E. L., D. J. Forrest., A. N. Suri, High energy gamma-ray radiation above $300 \mathrm{keV}$ associated with solar activity, in Solar Gamma-, $\mathrm{X}_{-}^{-}$, and EUV radiation, Ed. by S. R. Kane, p. 341 , Reidel, Dordrecht, Holliand, 1975:

Dickman, D. O., R. L. Morse, and C. W. Nielson, Numerical simulation of axisymmetric, collisionless, finite- $\beta$ plasma, Phys. Fluids 12, 1708, 1969.

Drake, J. F., and Y. C. Lee, Nonlinear evolution of collisionless and semicollisional tearing modes, Phys. Rev. Letts. 39, 453, 1977.

Forrest, D. J., E. L. Chupp, J. M. Ryan, C. Reppin, E. Rieger, G. Kanbach, K.

Pinkau, G. Share and G. Kinzer, Evidence for impulsive ion acceleration during the 0312UT flare of 1980 June 7, in Proceedings of the 17th International Cosmic Ray Conference, Paris, France, 1981, to be published.

Galeev, A. A., F. V. Coroniti, M, Ashour-Abdalla, Explosive tearing mode reconnection in the magnetospheric tail, Geophys. Res. Lett. 5, 707, 1978.

Gekelman, W., and R. L. Stenzel, Magnetic field line reconnection experiments, 2. Plasma parameters, J. Geophys. Res., 86, A2, 659, 1981.

Hamilton, J. E. M., J. W. Eastwood, The effect of a normal magnetic field component on current sheet stability, lanet. Space Sci. 30, 293, 1982. 210

Howard, R., and Z. Svetska, Development of a complex of activity in the solar corona, Solar Phys. 54, 65, 1977.

Katanuma, I., and T. Kamimura, Collisionless tearing instabilities, Phys. Fluids, 23, 2500, 1980.

Leboeuf, J. N., T. Tajima and J. M. Dawson, Magnetic x-points, islands coalescence and intense plasma heating, in Physics of Auroral Arc Formation, Ed. by S. I. Akasofu and J. R. Kan, p. 337, AGU, Washington, D. C., 1981.

Leboeuf, J. N., T. Tajima and J. M. Dawson, Dynamic x-points, Phys. Fluids, 25, $784,1982$. 
Parker, E. N., Cosmical magnetic Fields, Chapter 15, Clarendon Press, Oxford, 1979. Rutherford, P., Nonlinear growth of the tearing mode, Phys. Fluids 16, 1903, 1973.

Stenzel, R. L., and W. Gekelman, Experiments on magnetic field line reconnection, Phys. Rev. Letts. 42, 1055, 1979.

Sturrock, P. A., Flare models, in Solar Flares: A Monograph from Skylab Solar Workshop II, Ed. P. A. Sturrock, p. 411, Colorado Associated University Press, Boulder, 1980. Tajima, T., Tearing and Reconnection, in Fusion Energy - 1981 (Interkational Centre for for Theoretical Physics, Trieste, 1982), p. 403. International Atomic Energy Agency, Vienna, Austria, 1982.

Tajima, T., F. Brunel and J. Sakai, Loop coalescence in flares and coronal $\mathrm{x}$-ray brightening, Astophys. J., 258, L45, 1982.

Tajima, T., F. Brunel, J. Sakai, L. Vlahos and M. Kundu, The coalescence instability in solar flares, Proceedings of the IAU conference on Tnstable Current Systems in Astrophysical Plasmas, Ed. by M. Kundu, IAU, 1985. P 197. (North-Holland) Terasawa, T., Numerical study of explosive tearing mode instability in one-component plasmas, J. Geophys. Res. 86, 9007, 1981.

Wu, C. C., J. N. Leboeuf, T. Tajima and J. M. Dawson, Magnetic islands coalescence and intense plasma heating, University of California at Los Angeles, Plasma Physics Group Report No. PPG-551, 1981. 511

This paper was presented on Chapman Conference on Magnetic Reconnection held at the Los Alamas National Laboratory, U.S. A. October 3-7, 1983. 